Trauma Berufskrankh 2005 · 7[Suppl 1]:S45-S50 DOI 10.1007s10039-004-0985-3

Online publiziert: 25. Januar 2005

(c) Springer Medizin Verlag 2005

Ch. Josten · B. Marquaß

Abteilung für Unfall-, Wiederherstellungs- und Plastische Chirurgie,

Universitätsklinikum, Leipzig

\title{
Frakturen der proximalen Tibia
}

\author{
Marknagel vs. LISS
}

D ie proximale Fraktur der Tibia kann bei dem älteren osteoporotischen Patienten durch ein geringes Trauma verursacht werden. Häufiger sind jedoch hochenergetische Traumen bei jüngeren Patienten. Dies zeigt sich auch im Altersdurchschnitt, der für die proximale Tibiafraktur bei 49,8 Jahren liegt [4]. Aufgrund der fehlenden Weichteildeckung der anteromedialen Seite kommt es neben einem hohen Anteil an offenen Frakturen, welcher von Court-Brown et al. [5] mit 23,5\% angegeben wurde, auch häufiger zu Wundheilungsstörungen und zu einer mitunter verzögerten Frakturheilung, bedingt durch einen reduzierten periostalen Blutfluss. Der Schweregrad der Weichteilschädigung korreliert mit dem der Fraktur. Durch Verletzungen der Metaphyse oder Schädigung von Weichteilen kann es zu einer Einblutung in die Muskellogen des Unterschenkels kommen. Enge klinische Kontrollen sind daher auch postoperativ unerlässlich, um rechtzeitig die Entwicklung eines Kompartmentsyndroms zu erkennen.

\section{Osteosynthese}

Die Frakturheilung bzw. die Gefahr einer Pseudarthrose ist abhängig vom Grad der Stabilität einer Osteosynthese. Dieser variiert zwischen absolut und relativ stabil, je nachdem ob die Osteosynthese eine Kompression oder Schienung bewirkt. In
Abhängigkeit vom gewählten Verfahren kommt es zu einer direkten oder indirekten Knochenbruchheilung.

Die Art der Fraktur spielt bei der Wahl der Osteosynthese die entscheidende Rolle. Eine Fragmentverschiebung führt bei einfachen Frakturen zu einer hohen Relativverschiebung der beiden Frakturenden. Liegt hingegen eine Trümmerfraktur mit mehreren kleineren Fragmenten vor, ist die Relativverschiebung aufgrund der Vielzahl der Fragmente deutlich geringer. Um das Pseudarthroserisiko gering zu halten, sollte daher eine einfache Fraktur präzise reponiert und stabil fixiert werden. Bei Frakturen mit Trümmerzone sind hingegen Mikrobewegungen bewusst erwünscht, um eine indirekte Knochenheilung durch Kallusbildung anzuregen.

Der operativen Versorgung geht eine genaue Diagnostik und Einteilung anhand der AO-Klassifikation voraus. Offene Verletzungen werden nach Gustilo und Anderson eingeteilt. Die Wahl des Osteosyntheseverfahrens hängt sowohl vom Frakturverlauf als auch vom begleitenden Weichteilschaden ab. Bei einer isolierten Tibiafraktur ist bei gleichzeitig bestehender Fibulafraktur die Gefahr einer Achsabweichung gegeben. Im Fall einer kompletten Unterschenkelfraktur konnten gute klinische Ergebnisse auch ohne Fibulaverplattung erreicht werden [9]. Die am häu- figsten Verwendung findenden Verfahren bei der proximalenTibiafraktur sind

- der von proximal eingebrachte Marknagel und

- die Plattenosteosynthese,

wobei aufgrund der verbesserten Biomechanik zunehmend winkelstabile Implantate verwendet werden (z. B. LISS, Fa. Synthes).

\section{Marknagel}

\section{Frakturtyp}

Der intramedulläre Nagel hat sich als das Verfahren der Wahl bei Schaftfrakturen etabliert. Als biomechanisch ideales Osteosyntheseverfahren bietet er bei bestehender Stabilität die Möglichkeit der Dynamisierung. Besondere Indikationsstellungen sind langstreckige Frakturen und die gleichzeitige Osteosynthese von 2-Etagen-Frakturen. Der intramedullären Technik sind jedoch bei kleinen proximalen Fragmenten Grenzen gesetzt, da von der Nagelkonfiguration her eine sichere Verankerung ab einer bestimmten Fragmentgröße nicht weiter möglich ist. Hier erlauben anatomisch vorgeformte Nägel mit einer maximalen proximalen Verankerung (z. B. Sirus-Nagel, Fa. Zimmer) die Versor- 


\section{Neue Osteosynthesetechniken}
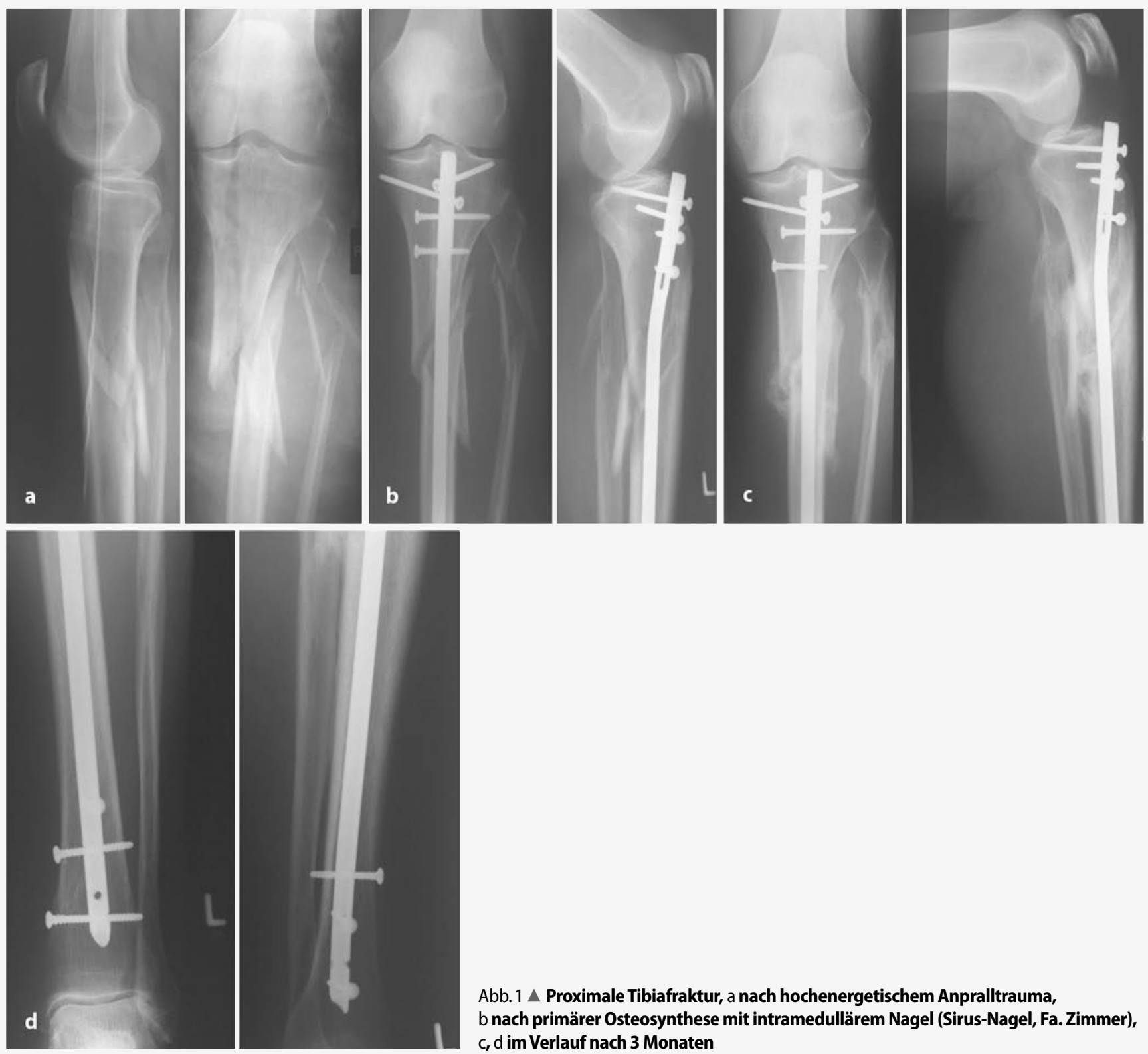

Abb. $1 \Delta$ Proximale Tibiafraktur, a nach hochenergetischem Anpralltrauma, b nach primärer Osteosynthese mit intramedullärem Nagel (Sirus-Nagel, Fa. Zimmer), c, d im Verlauf nach 3 Monaten
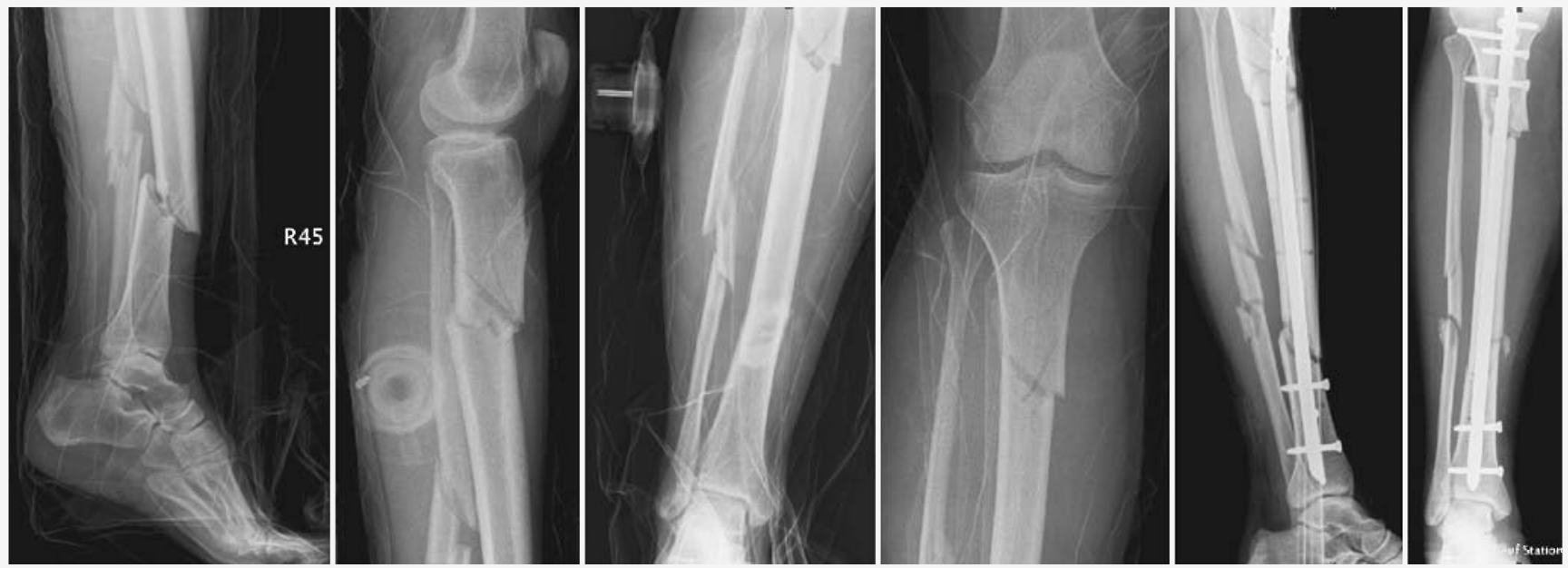

Abb.2 $\Delta$ Intramedulläre Osteosynthese nach 2-Etagen-Fraktur mit Sirus-Nagel 


\section{Zusammenfassung $\cdot$ Abstract}

gung von Frakturen bis zu 4/5 der distalen Tibia (• Abb. 1, 2).

\section{Weichteilsituation}

Aufgrund der intramedullären Lage bleibt die Belastung der Weichteile minimal, wodurch der Nagel als ideales Implantat bei kritischer Weichteilsituation zu sehen ist.

\section{Fallstricke}

Die Nachteile des Nagels zeigen sich bei der Versorgung besonders proximal gelegener Frakturen. Hier kann es zu Achsabweichungen und Rotationsfehlern kommen. Aus einem zu weit distal oder anterior gewählten Eintrittspunkt resultiert eine vermehrte Anteversion des distalen Fragments. Eine Valgisierung der Tibia entsteht bei zu weit medial gewähltem Eintrittspunkt des Nagels. Zudem neigt das proximale Fragment dazu, durch den einwirkenden Muskelzug nach dorsal und in den Valgus abzukippen $[6,7]$. Zudem muss differenziert werden, ob ein Achsfehler aufgrund ungenügender Reposition primär bestand und durch das Einbringen des Nagel lediglich fixiert wurde - anders als bei Schaftfrakturen findet bei proximalen Frakturen keine Korrektur durch das Implantat statt - oder ob es sekundär postoperativ durch Belastung zu einer Abweichung kam. Freedmann u. Johnson [6] fanden bei proximalen Tibiafrakturen Achsabweichungen nach intramedullärer Osteosynthese von 58\%. Besonders bei kleineren proximalen Fragmenten ist die Gefahr der sekundären Dislokation und Lockerung unter Belastung erhöht, wodurch die Bedeutung einer festen proximalen Verankerung betont wird.

\section{LISS}

\section{Frakturtyp}

Aufgrund der besseren Weichteildeckung wird die Platte in den meisten Fällen auf der lateralen Tibiaseite über einen anterolateralen Zugang eingebracht. Ein zweiter mediodorsaler Zugang muss in der Regel aufgrund der Winkelstabilität nicht gewählt werden. Selten kann in Abhängigkeit vom Frakturverlauf auch primär eine mediale Abstützung erfolgen [8].

Trauma Berufskrankh $2005 \cdot 7[$ Suppl 1]:S45-S50

DOI 10.1007/s10039-004-0985-3

c) Springer Medizin Verlag 2005

Ch. Josten · B. Marquaß

\section{Frakturen der proximalen Tibia. Marknagel vs. LISS}

\section{Zusammenfassung}

Proximale Tibiafrakturen sind häufig Hochrasanztraumen, die bei einer oft schlechten Weichteildeckung vielfach als offene Frakturen imponieren. Ihre chirurgische Versorgung stellt trotz moderner Osteosyntheseverfahren, aufgrund einer Dislokation durch nach proximal und distal wirkende Muskelzüge, einer kompromittierten Weichteilsituation sowie der Gefahr von Achsfehlern weiterhin eine chirurgische Herausforderung dar. Zunehmend finden moderne Implantate wie der anatomisch geformte intramedulläre Nagel und die winkelstabile Plattenosteosynthese Ver-

\section{Proximal tibial fractures. Intramedullary nailing vs LISS}

\section{Abstract}

Most proximal tibial fractures are high-energy traumata. They often give the impression of open fractures because of poor soft tissue conditions. In spite of modern osteosynthetic implants, the surgical treatment is still a challenge, some of the problems being dislocation of the proximal fragment resulting from opposing muscle forces, a difficult soft issue situation and the danger of malalignment. Such modern implants as anatomically shaped intramedullary nails and fixed-angle plates are increasingly used for fixation. The two methods are characterized by differ- wendung. Die Methoden sind durch unterschiedliche biomechanische Eigenschaften gekennzeichnet und erfordern differenzierte Operationstechniken. In diesem Beitrag werden Vor- und Nachteile des jeweiligen Implantats anhand der Literatur und eigener Nachuntersuchungen dargestellt, miteinander verglichen und eine Indikationsempfehlung aufgezeigt.

\section{Schlüsselwörter}

Proximale Tibia · Fraktur · Marknagel .

LISS - Osteosynthese ent biomechanical behavior and demand specific operation techniques. This article describes the advantages and disadvantages of each and compares them in the light of the current literature and of the authors' own observations at follow-up examinations. Finally, recommendations on how to recognize when each is indicated are given.

\section{Keywords}

Proximal tibia - Fracture .

Intramedullary nail · LISS · Osteosynthesis 


\section{Neue Osteosynthesetechniken}
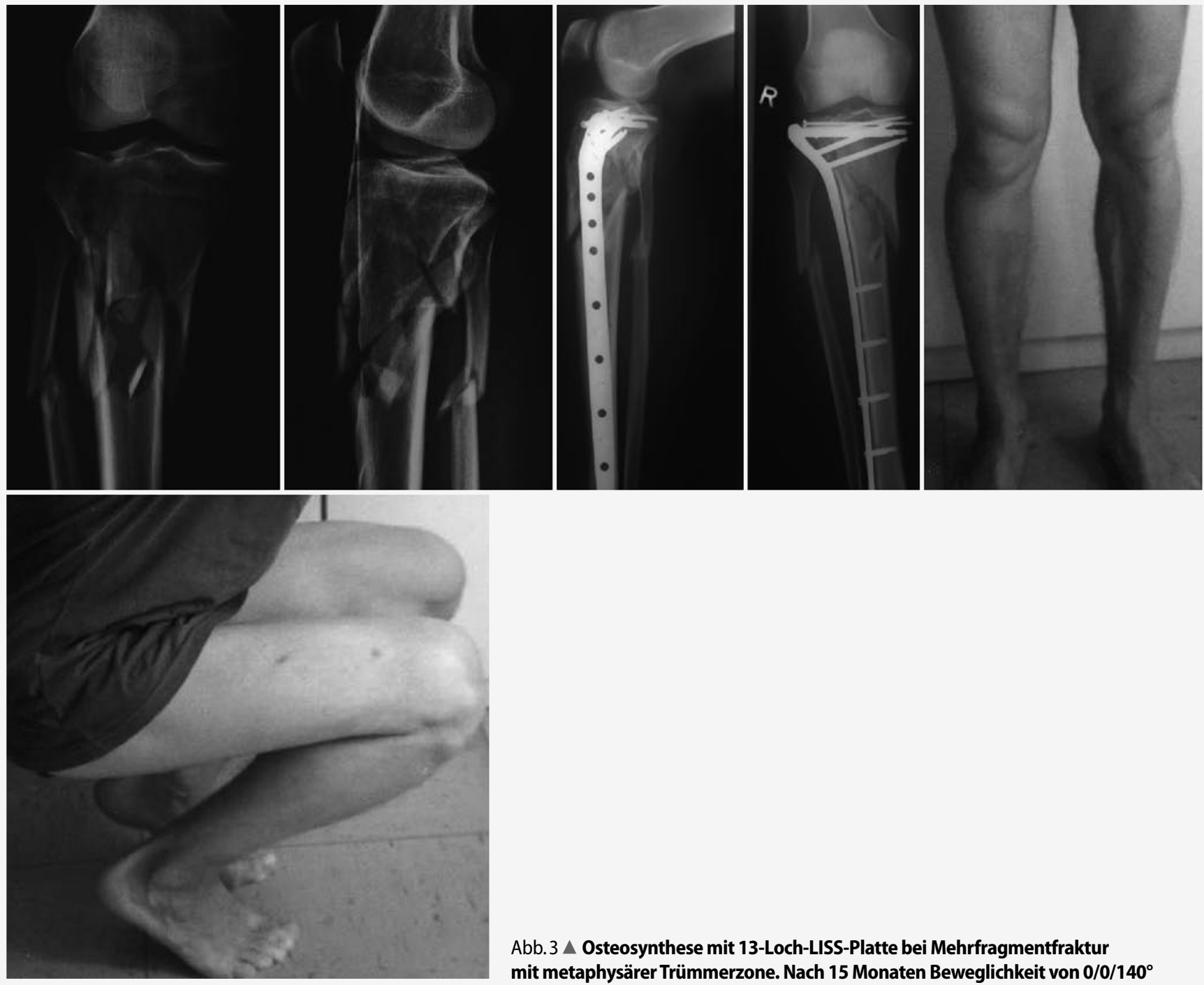

Abb. $3 \Delta$ Osteosynthese mit 13-Loch-LISS-Platte bei Mehrfragmentfraktur mit metaphysärer Trümmerzone. Nach 15 Monaten Beweglichkeit von 0/0/140

Die Domäne des LISS liegt in

- der sicheren Verankerung von kleinen proximalen Fragmenten,

- der Versorgung von Tibiakopffrakturen und

- der Osteosynthese osteoporotischer Frakturen (• Abb. 3, 4).

\section{Weichteilsituation}

Das LISS stellt ein nur begrenzt minimalinvasives Verfahren dar. Es resultiert eine im Vergleich zum Marknagel höhere Invasivität mit nachfolgender vermehrter Schädigung der die Fraktur umgebenden Weichteile durch das Durchschieben der Platte. Um eine erhöhte Infektionswahrscheinlichkeit zu vermeiden, ist eine möglichst Gewebe schonende Vorgehensweise unabdinglich.

In einer evidenzbasierten Übersichtsarbeit fanden Bhandari et al. [2] für den in- tramedullären Nagel eine mit 2,5\% geringere Infektionsrate im Vergleich zu Plattenosteosynthesen (14\%) oder externer Fixation (8\%). Für LISS fanden Cole et al. [1] in einer Untersuchung an 77 Patienten eine Infektionsrate von $6,5 \%$ (oberflächliche und tiefe Infektionen). Neben der erhöhten Infektionsrate stellen Weichteilinterponate und die Gefahr einer Läsion der N. peronaeus superficialis weitere Komplikationen dar.

\section{Fallstricke}

Die Frakturreposition erfolgt indirekt und vor dem Einbringen der Platte. Die Folge sind gerade zu Beginn der hohen individuellen Lernkurve verlängerte Durchleuchtungszeiten und ein erhöhtes Risiko von Achsfehlstellungen. Die Kombination von LISS und AO-Distraktor konnte diese Schwächen verbessern, und sowohl die Durchleuchtungszeiten als auch die Fehlstellungen ließen sich reduzieren [1]. Dennoch bleibt das Risiko von Achsfehlstellung nach LISS-Osteosynthese erhöht.

In einer eigenen retrospektiven Studie untersuchten wir in einem 3-Jahres-Zeitraum 27 Patienten mit einem durchschnittlichen Alter von 55 Jahren (• Abb. 5, 6). Bei einem überwiegenden Anteil an CFrakturen wurden 4 primär und 16 postprimär nach temporärer Fixateur-externe-Anlage versorgt. In 7 Fällen erfolgte die Osteosynthese als sekundäre Revision. Nach einem durchschnittlichen Followup von 14,3 Monaten fanden sich Achsabweichungen von $>5^{\circ}$ in 7 Fällen. Im Rasmussen-Score gaben $86 \%$ der Patienten gute bis sehr gute Werte an.Eine jüngste Untersuchung konnte besonders in der Sa- 

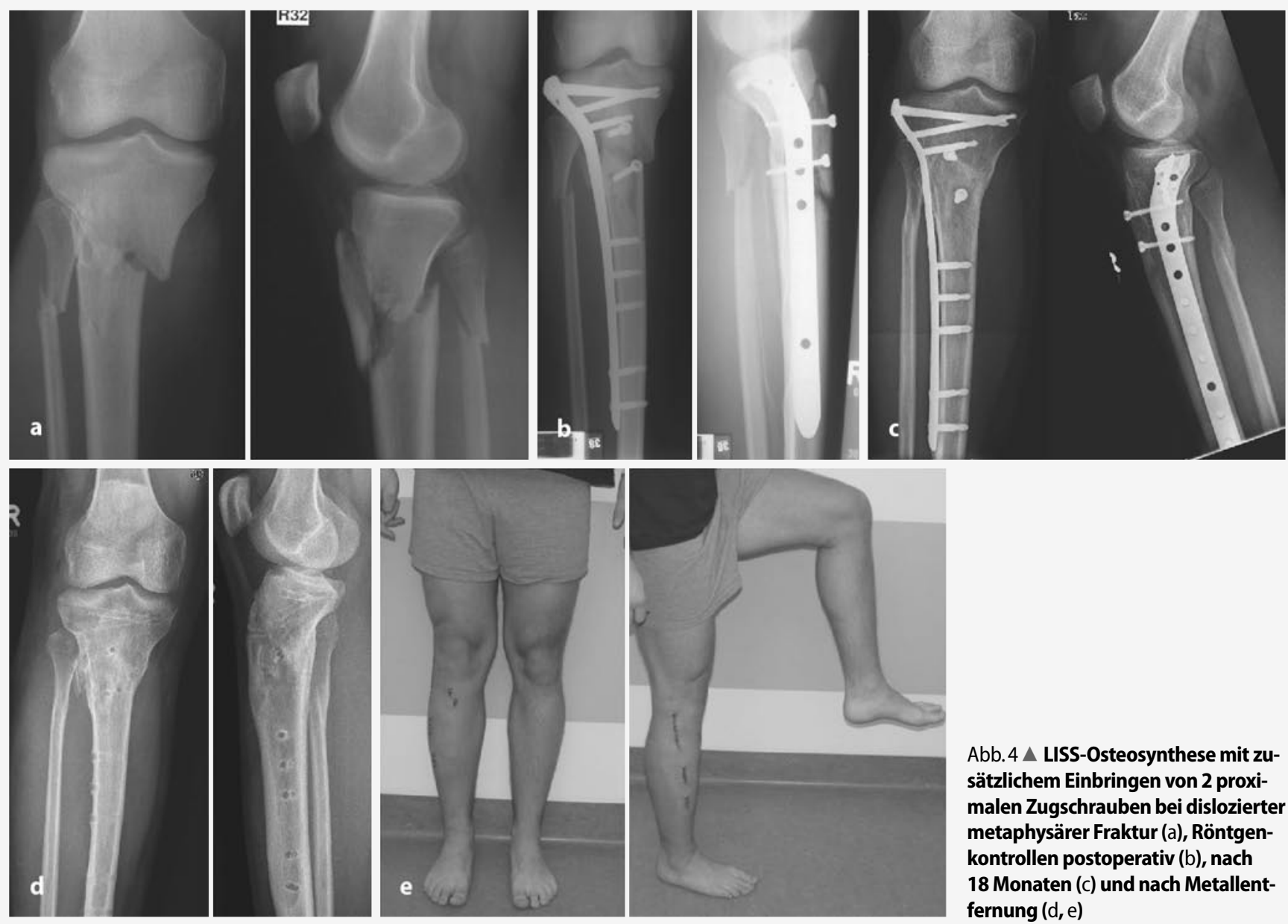

Abb. 4 \ LISS-Osteosynthese mit zusätzlichem Einbringen von 2 proximalen Zugschrauben bei dislozierter metaphysärer Fraktur (a), Röntgenkontrollen postoperativ (b), nach 18 Monaten (c) und nach Metallentfernung $(d, e)$

3

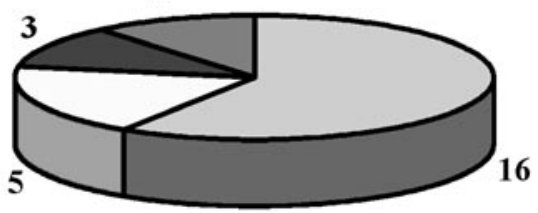

$\square$ geschlossen $\square$ II $^{\circ}$ offen

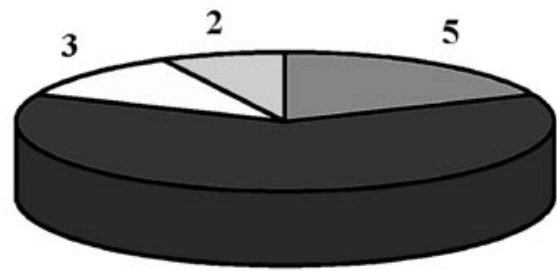

17

41A $\square$ 41C $\square 42 \square$ Komb.41/42
Abb. $6<$ Frakturverteilung, AO-Klassifikation

Abb. 5 Verteilung der Weichteilschäden bei 27 Patienten nach Gustilo und Anderson

gittalebene Flexionsabweichungen von $>5^{\circ}$ nachweisen [3].

\section{Diskussion}

Die proximale Tibiafraktur nimmt eine Sonderstellung in der chirurgischen Frakturversorgung ein und stellt aufgrund der Kombination von häufig kritischer Weichteilsituation und schwieriger Reposition der proximalen Fragmente, nicht zuletzt durch nach proximal und distal wirkende Muskelzüge, eine chirurgische Herausforderung dar.
Wie dargestellt wurde, bieten LISS und der intramedulläre Nagel unterschiedliche Vor- und Nachteile. Überlegt eingesetzt ergänzen sich beide Systeme in der Behandlung proximaler Tibiafrakturen. Jedem Verfahren kommt bei einem fließendem Übergang zwischen den Methoden eine feste Position im Therapiealgorithmus zu.

Wesentliche die Wahl der Osteosynthese beeinflussende Faktoren sind

- Frakturlokalisation (metaphysär/diaphysär),
- Frakturart (einfache Fraktur/Mehrfragmentfraktur/offen/geschlossen) und

- bestehende Weichteilschädigung.

Des Weiteren bietet LISS im osteoporotischem Knochen eine vermehrte Stabilität. Erst die Entwicklung von anatomisch geformten Marknägeln mit der Möglichkeit einer maximalen proximalen Verankerung erlaubt auch den Einsatz intramedullärer Systeme bei osteoporotischen Frakturen. 
Der intramedulläre Nagel findet besonders bei langstreckigen Frakturen der distalen 4/5 der Tibia Anwendung. Ein hoher traumatischer Weichteilschaden oder eine offene Fraktur begünstigen die Indikationsstellung. Zur Vermeidung von primären oder sekundären Achsabweichungen muss besonders bei proximal gelegenen Frakturen auf eine achsgerechte Reposition, ein regelrechtes Einbringen des $\mathrm{Na}-$ gels und eine maximale proximale Verankerung hoher Wert gelegt werden.

Das LISS sollte besonders bei Frakturen der proximalen $1 / 5$ der Tibia und Tibiakopffrakturen verwendet werden. Das kurze gelenknahe Fragment kann aufgrund der Winkelstabilität trotz ungünstiger Belastungshebel fixiert werden. Ein hoher Weichteilschaden kann die definitive Frakturversorgung zeitlich verzögern und den Wechsel auf ein intramedulläres System, sofern es die Frakturlokalisation zulässt, oder eine temporäre Ruhigstellung mit einem Fixateur externe notwendig machen.

\section{Korrespondierender Autor}

\section{Dr. B. Marquaß}

Abteilung für Unfall-, Wiederherstellungs- und Plastische Chirurgie, Universitätsklinikum, Liebigstraße 20, 04103 Leipzig

E-Mail: Bastian.Marquass@medizin.uni-leipzig.de

Interessenkonflikt: Der korrespondierende Autor versichert, dass keine Verbindungen mit einer Firma, deren Produkt in dem Artikel genannt ist, oder einer Firma, die ein Konkurrenzprodukt vertreibt, bestehen.

\section{Literatur}

1. Babst R, Hehli M, Regazzoni P (2001) LISS-Traktor - Kombination des „less invasive stabilization systems" (LISS) mit dem AO-Distraktor für distale Femur- und proximale Tibiafrakturen. Unfallchirurg 104: 530-535

2. Bhandari M, Audige L, Ellis T et al. (2003) Operative treatment of extra-articular proximal tibial fractures. J Orthop Trauma 17: 591-595

3. Cole PA, Zlowodzki M, Kregor PJ (2004) Treatment of proximal tibia fractures using the less invasive stabilization system: surgical experience and early results in 77 fractures. J Orthop Trauma 18: 528-35

4. Court-Brown CM, McBirnie J (1995) The epidemiology of tibial fractures. J Bone Joint Surg $\mathrm{Br} 77$ : 417-421

5. Court-Brown CM, Rimmer S, Prakash U et al. (1998) The epidemiology of open long bone fractures. Injury 29: 529-534

6. Freedmann EL, Johnson EE (1995) Radiographic analysis of tibial fracture malalignment following intramedullary nailing. Clin Orthop 315: 25-33
7. Henley MB, Meier M, Tencer AF (1993) Influences of some design parameters on the biomechanics of the unreamed tibial intramedullary nail. J Orthop Trauma 7: 311-319

8. Krettek C, Gerich T, Miclau T (2001) A minimally invasive medial approach for proximal tibial fractures. Injury [Suppl] 1: SA4-13

9. Williams TM, Marsh JL, Nepola JV et al. (1998) External fixation of tibial plafond fractures: is routine plating of the fibula necessary? J Orthop Trauma 12: 16-20
TraumaundBerufskrankheit. springer.de

"Online First"

Trauma und Berufskrankheit informiert Sie regelmäßig über praxisrelevante Themen und die neuesten wissenschaftlichen Erkenntnisse lhres Fachgebiets. Um die Publikationszeiten zu verkürzen und Ihnen einen noch schnelleren Zugriff auf aktuelle Beiträge zu ermöglichen, heißt es ab sofort „Online First“.

Unter dem Begriff "Archiv" finden Sie auf der Zeitschriftenhomepage den Zugang zum elektronischen Volltextarchiv Ihrer Zeitschrift. Hier stehen Ihnen unter "Online First" aktuelle Beiträge bereits vor dem Erscheinen der gedruckten Ausgabe als HTML-Version zur Verfügung. Jeder „Online-First"-Beitrag ist durch eine elektronische Identifizierungsnummer, den Digital Object Identifier (DOI), eindeutig gekennzeichnet, sofort zitierfähig und suchbar.

Als besonderen Zusatznutzen enthält die HTML-Version Referenz-Verlinkungen, die Sie direkt zu den Originalzitaten auf SpringerLink, ChemPort und Pubmed führen. Ein Service, der Ihnen ein Mehr an Informationen in Ihrem Fachgebiet sichert.

Haben Sie noch Fragen oder Anmerkungen?

Wir freuen uns über Ihre E-Mail an springerlink@springer-sbm.com Ihre Redaktion Fachzeitschriften Medizin/Psychologie 